\title{
Historia de Acta Médica Colombiana
}

\section{History of Acta Medica Colombiana}

\author{
Paulo Emilio Archila • Bogotá D.C. (Colombia)
}

En el libro de Carlos Arturo Hernández, Enrique Ardila y María Nelly Niño de Arboleda titulado Medicina Interna en Colombia, editado por la ACMI ${ }^{\circledR}$ en el año 2006, me correspondió escribir el capítulo Los Oficios de Acta Médica Colombiana en la sección Publicaciones de la ACMI ${ }^{\circledR}$ (1), reproducido en el suplemento de la revista del volumen 34 de 2009, editado como homenaje a la Asociación Colombiana de Medicina Interna en sus 50 años de existencia (2). El capítulo contiene los primeros 30 años de historia de Acta Médica Colombiana, rememorando todos los personajes que hicieron la revista así como una selección de los mejores artículos de opinión, filosofía, ciencia, y conferencias magistrales institucionales, que también se reprodujeron en el suplemento para poner en evidencia la evolución del pensamiento científico y gremial de la medicina interna colombiana, dejándola al alcance de las nuevas generaciones como prueba histórica de la existencia de la $\mathrm{ACMI}^{\circledR}$.

En esta ocasión me han solicitado que escriba de nuevo sobre la historia de AMC; no es mi intención repetir, quiero en lugar de hacer una enumeración histórica, relatar cuál ha sido la filosofía y el trabajo para obtener nuestra publicación.

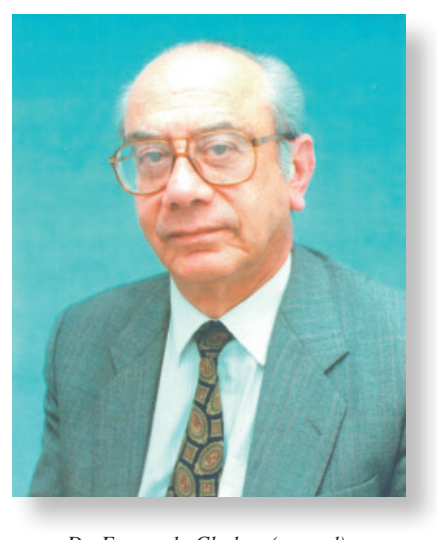

Dr. Fernando Chalem (q.e.p.d)

La razón de ser de una revista científica es la publicación de la investigación, basados en el principio enunciado por Dora Inés Molina en su conferencia titulada Impacto de la investigación clínica en el desarrollo de un país (3), reproducida en este suplemento, quien sostiene que la ciencia no podría existir sin escribir. Se publica no solo la investigación científica en todos sus pasos, sino que se publican resultados de la medicina basada en la evidencia con toda su metodología, pues estas publicaciones contribuirán a su vez a otras investigaciones basadas en la literatura.

Consciente de esa realidad, el Dr. Fernando Chalem presentó en noviembre de 1975 a la Junta Directiva Nacional de Asociación Colombiana de Medicina Interna el proyecto de la revista de la ACMI $^{\circledR}$, como un ente autónomo en cuanto a su independencia editorial y financiera, pero con la auditoría de la Asociación en su calidad de propietaria de la que se llamaría Acta Médica Colombiana. El primer número salió a circulación en marzo de 1976, con la invaluable colaboración de los doctores Ernesto Laverde y Jimeno Ramírez como Asistentes del Editor Fernando Chalem (4). Jorge Escandón fue el Jefe de Redacción hasta 1997 cuando asumió el cargo de Editor General hasta 1998 cuando renunció por conflictos con las directivas de la Asociación. Durante esos 22 años la revista contó con la colaboración de innumerables socios de $\mathrm{ACMI}^{\circledR}$ en calidad de comité editorial, consejo consultivo, correctores de prueba etc. Esa constituyó la que yo llamaría primera generación de Acta Médica.

Hasta entonces se trabajaba sin ayudas tecnológicas: los trabajos eran leídos y considerados por el comité consultivo quien se encargaba de evaluar la calidad del contenido para ser luego aprobados por el comité editorial, quien debía verificar si se ajustaba a las normas editoriales y éticas de la revista, pasaban a corrección de estilo, por correctores internistas jóvenes quienes deberían velar para que las pruebas recibidas a máquina fueran

Paulo Emilio Archila, MD FACP: Editor Emérito Acta Médica Colombiana. Bogotá, D.C. (Colombia).

E-mail: pauloearchila@gmail.com 
corregidas de acuerdo con sus observaciones manuales en la casa editora y verificarían finalmente las pruebas azules de los textos que serían impresos. Este era un proceso muy lento y engorroso dejando la responsabilidad del

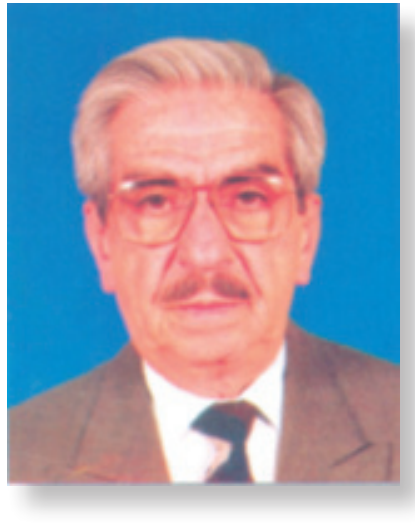

Dr. Jorge Escandón (q.e.p.d) contenido y forma de la revista exclusivamente en manos del Editor (5). Hubo momentos críticos en los cuales no había material científico suficiente para una publicación y debían los integrantes de la revista escribir revisiones de tema con carácter urgente para poder cumplir con la meta editorial y los contratos de publicidad. Afortunadamente, con el progreso de la revista y su creciente divulgación comenzaron a sobrar artículos, hasta el punto en el cual el comité editorial decidió incrementar la frecuencia de trimestral a bimestral a partir de 1982 y modificar la forma a dos columnas por una versión a tres columnas que ahorraría espacio para tener una revista financiable y así permaneció hasta 1998 cuando la crisis financiera del país limitó los ingresos de la revista por reducción del aporte publicitario y la explosión de revistas de subespecialidades, disminuyó el número de trabajos puestos a consideración de nuestro comité. Entonces regresó la revista a su edición trimestral a partir de 2002.

Para esta primera generación fue mucho el trabajo desarrollado para llevar Acta Médica al nivel de calidad y transparencia que tenía a finales del siglo XX. Tuvieron que aprender de algo que no estaba escrito y ese era el reto de todas las publicaciones mundiales. Ya desde 1978 se estaba intentando uniformidad en las publicaciones con la aparición del Comité Guía Internacional para Requisitos Uniformes para manuscritos sometidos a revistas biomédicas, conocido como el acuerdo de Vancouver, al cual se adhirió Acta Médica en 1982 e inició su implementación. Este se transformó en el Comité Internacional de Editores de Revistas Médicas y mantiene actualizados los requisitos uniformes, ajustándolos a las crecientes facilidades de la tecnología actual de la comunicación. La última versión aparece publicada en este suplemento. Seguros de que la aplicación de las nuevas normas era suficiente y Acta Médica había clasificado en el primer lugar del Publindex de Colciencias, tanto el Dr. Chalem como el Dr. Escandón durante sus períodos sometieron la revista al proceso de indización en la Biblioteca Nacional de los Estados Unidos para ser incluida en el Med-line o Index Medicus. La revista no clasificó así como fue descabezada del Publindex, pues los requisitos de indización tanto nacional como internacional se hicieron cada vez más estrictos. En esa etapa asumí yo el cargo de Editor en 1999, sin recibir ninguna instrucción de nadie, dispuesto a aprender y a mejorar, puesto que traía la experiencia de diez años como editor de la Revista Colombiana de Gastroenterología con los mismos tropiezos que AMC.

Afortunadamente la mayoría de miembros del Comité Editorial que tenía el Dr. Escandón aceptó acompañarme en el nuevo período que yo llamo la segunda generación de Acta Médica Colombiana, correspondiente al siglo XXI y aportaron la experiencia necesaria para que la publicación no claudicara.

El objetivo era mantener el nivel de calidad de la revista, implementando los cambios obligatorios para que una publicación biomédica pudiera ser aceptada en todos los índices nacionales e internacionales posibles, logrando un factor de impacto que no solo la caracterizara como un punto de referencia obligatoria, sino que fuera lo suficientemente atractiva para mantener el interés en los patrocinadores económicos y mantener su independencia financiera (6).

Nuestro primer acercamiento con la era digital fue la edición de un CD-Rom con todos los resúmenes de los artículos publicados desde 1976 en conmemoración de los 25 años de la revista.

Se tecnificó la página web iniciada por el Dr. Escandón para ampliar su visibilidad, pero no había ninguna experiencia colombiana en publicaciones biomédicas en el ciberespacio.

Los requerimientos de Colciencias eran cada vez más estrictos y cambiantes, pues ellos tampoco tenían experiencia, de modo que decidimos trabajar en la creación de una base de datos colombiana con la asesoría del Bireme del Brasil, creando con las demás revistas medicas nacionales la base Scielo-Colombia. Esta llenaría las expectativas de las demás bases de datos y aprenderíamos a marcar adecuadamente nuestros artículos y a organizar las directivas de la revista con todos los requisitos de Vancouver. Esto llevó varios años pero logramos no solo ingresar a Lilacs, Latindex, Scielo, Publindex y Redalyc, sino quedamos en capacidad de optar por clasificar en las bases de datos de Estados Unidos y Europa Medline, Scopus, ISI y así obtener el mayor puntaje (6). Con el ingreso a Scielo logramos la publicación en texto completo de todos los artículos desde la creación de ese portal, además de lo que ya estábamos publicando en nuestra propia página web,

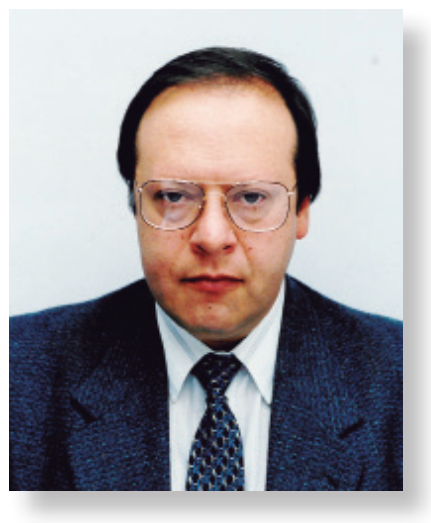

Dr. Paulo Emilio Archila asociados a la de $\mathrm{ACMI}^{\circledR}$.

¿Por qué fue tan importante ese cambio de visión hacia las bases de datos? Nos llevó a internacionalizar la revista aumentando notablemente la cantidad y calidad de trabajos puestos a nuestra consideración. 
Un aspecto muy importante en toda esa década fue la obligación de revisión por pares de todos los trabajos tanto de investigación, como de revisión y de presentación de casos. Es lo que demanda más esfuerzos, no solo por la dificultad de encontrar pares académicos que cumplan con los estrictos requisitos de Colciencias y de Vancouver, sino por lograr que ellos acepten su encargo con la rapidez necesaria para no desactualizar los trabajos. Los autores han aceptado las recomendaciones y el sistema ha logrado servir como una fuente de enseñanza para el internista colombiano en cómo publicar. Esto tiene dos connotaciones mayores: la metodología de la investigación así como su escritura deben ser perfectas, transparentes, enunciando los conflictos de interés para poder decidir la conveniencia de su publicación y deben seguir los paráme- tros de los Comités de Ética de las instituciones donde se llevan a cabo, para estar completamente dentro del marco legal. En este suplemento se publican varios artículos en ese sentido (6-7).

Otra dificultad que hemos encontrado permanentemente es la veracidad y autenticidad de las referencias bibliográficas y la prevención del plagio. Creo que hemos avanzado positivamente en ese aspecto.

A partir de 2010 asumió el Dr. Eugenio Matijasevic el cargo de Editor general, con la ventaja de que él venía trabajando desde los tiempos del Dr. Chalem en Acta Médica Colombiana. Tiene el una visión clara de los problemas y los objetivos de una revista científica y los está aplicando con lujo de detalles.

Para mejorar aun más la visibilidad se contrató personal experto en bibliometría y en edición de revistas electrónicas y en el momento actual están todos los artículos publicados desde 1976 en la revista electrónica, consultable para todo el mundo en nuestra página web sin costo alguno, ya que Acta Médica Colombiana hace parte del grupo de Open Journal System (OJS), por medio del cual se dispone la lectura gratis a todos los interesados de todos los artículos, sin restricción alguna y se construyó el sistema de comunicación entre los

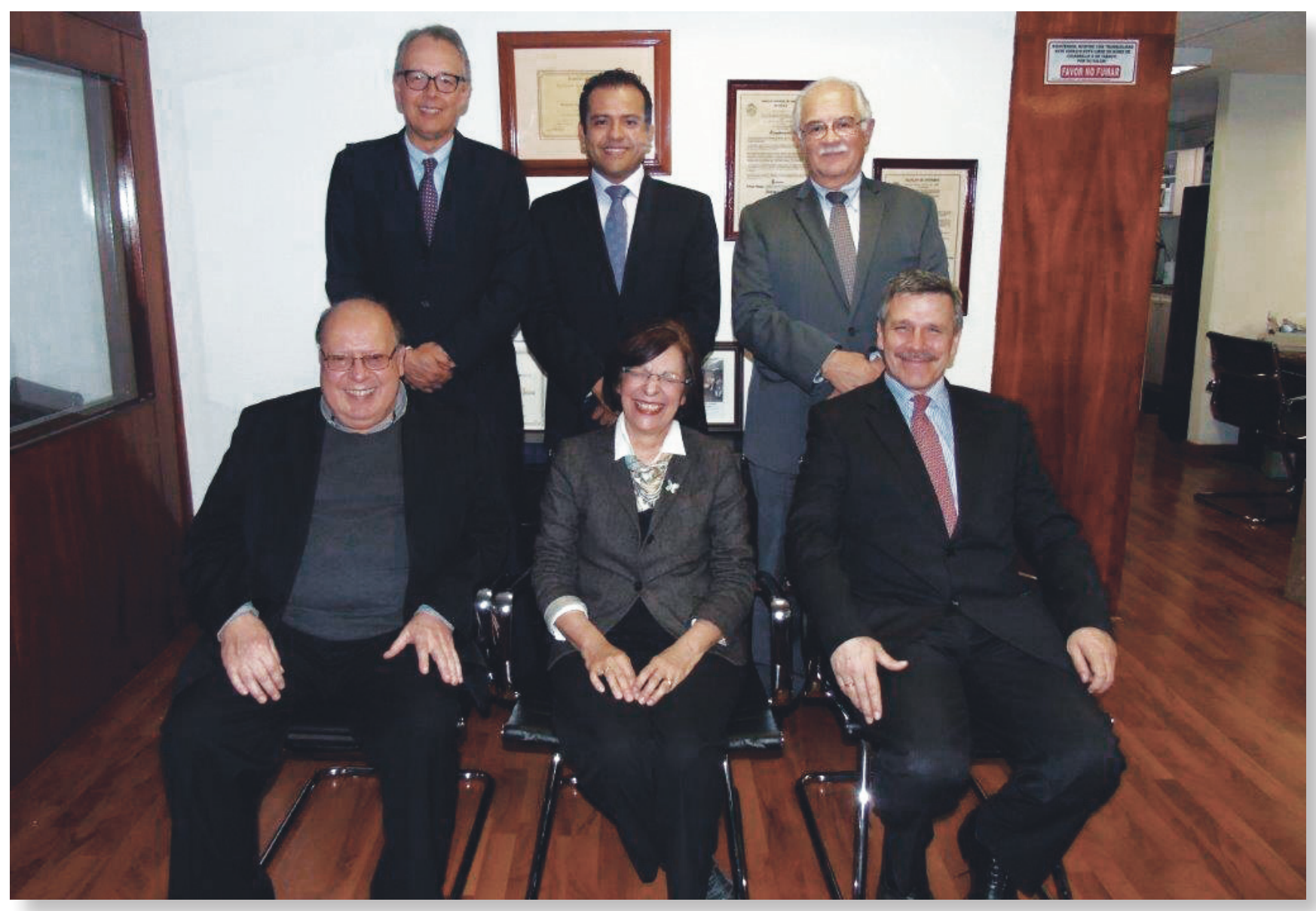

COMITE EDITORIAL AMC

Izquierda a derecha: de pie: Rodolfo Dennis, Eduardo Zúñiga, Roberto D’Achiardi; sentados: Paulo Emilio Archila, María Nelly Niño de Arboleda, Eugenio Matijasevic. 
autores, los editores, los pares académicos, los correctores, los diagramadores y la casa editorial a través de la red con un ahorro enorme de recursos y de energía, agilizando todo el proceso editorial. Es decir, pasamos de la máquina de escribir y las galeras de linotipo a la era totalmente digital de nuestra publicación.

Todo esto ha requerido gran esfuerzo de todo el equipo humano que interviene y un gran costo al haber digitalizado toda la revista desde su primer número. Disponemos de una tecnología que permite presentar los requisitos de indización anual a Colciencias y de refrendar fácilmente los internacionales actuales, y ojalá los futuros.

Para completar el avance tecnológico, a partir de este año se comenzó la publicación de revista electrónica a través del portal ebookmedico.com por convenio con la editorial Distribuna en la cual sale la revista en texto completo en español y en el futuro en inglés. Su acceso es de forma gratuita a través de la MDcard. Estas tarjetas están disponibles en las oficinas de la revista.

Esto no significa de manera alguna que haya desaparecido la publicación impresa ni el portal propio de AMC. Simplemente teniendo las tres formas aparte de nuestra inclusión en las bases de datos internacionales estaremos al día en lo que la tecnología pide para este siglo.

El papel de las Juntas Directivas de la ACMI ${ }^{\circledR}$ siempre ha sido favorable al grupo editorial de la Revista, respetando siempre la independencia editorial gracias a la firmeza de sus editores, con cambios en el aspecto financiero, obligados por la legislación actual, ya que al ser una dependencia de la Asociación, su contabilidad y financiación deben estar unificadas con la de ésta.
La labor del asesor editorial y del administrador de la página web, Doctores Wilson López y Luis Manuel Silva ha sido fundamental para los logros actuales y para mantenernos en un alto nivel de indización en el Publindex y en Scielo-Colombia, así como el incansable trabajo de nuestra coordinadora editorial Clara Ramírez en el contacto con los lectores, autores y suscriptores de AMC, con la organización de todos los manuscritos, su correcto y oportuno destino, las bases de datos internacionales, los autores y los pares académicos.

El grupo de Editores y el Comité Editorial siempre han respondido a los requerimientos del Editor General, Dr. Eugenio Matijasevic, quien se esmera en mantener la transparencia, ética y alto nivel científico amenizándonos además con sus excelentes editoriales de temática diversa.

Acta Médica Colombiana es una de las revistas de Medicina Interna más antiguas de Latinoamérica y la única razón para llegar a sus 40 años es su calidad y el amor con el cual todos hemos trabajado por ella.

\section{Referencias}

1. Archila PE. Publicaciones de la ACMI Los oficios de Acta Médica Colombiana. En: Hernandez CA, Ardila E, Niño de Arboleda MN. La Medicina Interna en Colombia. Bogotá ACMI® edición Chalver S.A. Zona Franca 1996: 183-191.

2. Archila PE. Publicaciones de la ACMI Los oficios de Acta Médica Colombiana. Acta Med Colomb 2009; 34 (supl) 141-146.

3. Molina de Salazar DI, Giraldo GC. Impacto de la investigación clínica en el desarrollo de un país. Acta Med Colomb 2012; 37:215-219.

4. Página bandera. Acta Med Colomb; 1976: III.

5. Matijasevic E. El toque de Midas. Acta Med Colomb 2010; 35:37-39

6. Matijasevic E. Acta Médica Colombiana y las fuerzas de producción social. Acta Med Colomb 2010: 35: 107-112.

7. Prieto P. Comités de ética en investigación con seres humanos: relevancia actual en Colombia. Acta Med Colomb 2011; 36:98-104. 